\title{
Efficient synchronization of Plasmodium knowlesi in vitro cultures using guanidine hydrochloride
}

\author{
Sutharinee Ngernna' ${ }^{1}$, Anongruk Chim-ong ${ }^{1}$, Wanlapa Roobsoong ${ }^{2}$, Jetsumon Sattabongkot ${ }^{2}$, Liwang Cui $^{3}$ \\ and Wang Nguitragool ${ }^{1 *}$ (D)
}

\begin{abstract}
Background: Long-term in vitro culture of blood stage Plasmodium parasites invariably leads to asynchronous parasite development. The most often used technique to synchronize Plasmodium falciparum culture is sorbitol treatment, which differentially induces osmotic lysis of trophozoite- and schizont-infected red blood cells due to presence of the new permeation pathways in the membranes of these cells. However, sorbitol treatment does not work well when used to synchronize the culture-adapted Plasmodium knowlesi A1-H.1 line.
\end{abstract}

Methods: A number of common solutes were tested in lieu of sorbitol for synchronization of P. knowlesi A1-H.1 ring stage.

Results: Guanidine hydrochloride was found to selectively lyse trophozoite- and schizont-infected red blood cells, yielding highly synchronous and viable rings.

Conclusions: A method for synchronization of $P$. knowlesi in human red blood cells was developed. Requiring only common laboratory reagents, this method is simple and should be applicable to most laboratory settings.

Keywords: Plasmodium knowlesi, Synchronization, Culture, Ring, Sorbitol, Guanidine hydrochloride, Enrichment

\section{Background}

Plasmodium knowlesi is a simian parasite that can also cause malaria in humans $[1,2]$. Whereas this parasite is prevalent in the Malaysian Borneo, its geographical range extends into the mainland Southeast Asia, Indonesia, and the Philippines [3]. Recently, the incidence of human infection of $P$. knowlesi in Malaysia and southern Thailand appears to be on the rise, despite the overall decline of malaria [3, 4]. The parasite is thus an emerging public health threat in affected areas.

Adaptation of $P$. knowlesi to continuous in vitro culture in human RBCs has opened doors to new possibilities to study this parasite $[5,6]$. As common for long-term

\footnotetext{
*Correspondence: wang.ngu@mahidol.edu

1 Department of Molecular Tropical Medicine, Faculty of Tropical Medicine, Mahidol University, 420/6 Ratchawithi Road, Ratchathewi, Bangkok 10400, Thailand

Full list of author information is available at the end of the article
}

Plasmodium culture, P. knowlesi culture loses synchronicity over time. Freshly thawed $P$. knowlesi culture becomes mixed stages within 4 to 5 days. However, a synchronized parasite culture is often needed in research, particularly when the aim is to examine stage-specific phenotypes, transcriptomes, and proteomes. Several synchronization methods developed for in vitro culture of Plasmodium falciparum have the potential for synchronization of in vitro P. knowlesi culture. These methods include magnetic separation to obtain mature trophozoites and schizonts $[7,8]$, selective lysis of trophozoites and schizonts to obtain rings [9], physical separation based on differential density [10] or sedimentation [11], and cold treatment to obtain rings [12, 13]. Indeed, density gradient centrifugation and magnetic separation have been used successfully to obtain tightly synchronized $P$. knowlesi culture [5, 6]. However, these methods are either time-consuming or require special equipment. For $P$. falciparum, lysis of trophozoite- and schizont-infected red 
blood cells (RBCs), first established in 1979, remains one of the most commonly used methods due to its high efficiency, simplicity and low cost [9]. This approach exploits the increased permeability of the infected RBCs due to the new permeation pathways (NPPs) to kill trophozoites and schizonts. NPPs allow sorbitol to enter the host cell, causing influx of water which leads to cell lysis. Because NPPs are active only in the host membrane at the trophozoite and schizont stages [14], the ring-infected RBCs are resistant to sorbitol treatment.

While the sorbitol method works well to synchronize P. falciparum, its application to the recently humanerythrocyte adapted line of P. knowlesi (A1-H.1) had limited success [15]. In this study, alternative solutes were explored and guanidine hydrochloride $(\mathrm{GuHCl})$ was found to selectively lyse trophozoite- and schizontinfected human RBCs to achieve synchronization of $P$. knowlesi culture.

\section{Methods}

\section{Plasmodium knowlesi in vitro culture}

The P. knowlesi strain A1-H.1 used in this study was from Dr. Robert W. Moon, Division of Parasitology, Medical Research Council National Institute for Medical Research, London. The parasite was cultured in complete medium ( $\mathrm{pH}$ 7.4) containing RPMI-1640 (Invitrogen), $5.96 \mathrm{~g} / \mathrm{L}$ HEPES, $2.3 \mathrm{~g} / \mathrm{L}$ sodium bicarbonate, $2 \mathrm{~g} / \mathrm{L}$ D-glucose, $0.292 \mathrm{~g} / \mathrm{L}$ L-glutamine, $0.05 \mathrm{~g} / \mathrm{L}$ hypoxanthine, $5 \mathrm{~g} / \mathrm{L}$ Albumax II (Invitrogen), $0.025 \mathrm{~g} / \mathrm{L}$ gentamycin sulfate, and 10\% (vol/vol) horse serum (Invitrogen). Human red blood cells (RBCs) were obtained from the Thai Red Cross at 50\% haematocrit and washed with RPMI-1640 containing $2 \mathrm{~g} / \mathrm{L}$ sodium bicarbonate, $5.94 \mathrm{~g} / \mathrm{L}$ HEPES, $2 \mathrm{~g} / \mathrm{L}$ D-glucose, and $0.025 \mathrm{~g} / \mathrm{L}$ gentamycin sulfate. RBCs were added to a $2-5 \%$ haematocrit and the culture was maintained in $75 \mathrm{~cm}^{2}$ flasks at $37{ }^{\circ} \mathrm{C}$ with gas mixture $\left(90 \% \mathrm{~N}_{2}, 5 \% \mathrm{CO}_{2}\right.$, and $\left.5 \% \mathrm{O}_{2}\right)$.

\section{Initial testing of different solutes for synchronization of ring-stage parasites}

Four solutes were tested to synchronize A1-H.1 parasite to the ring stage: sorbitol, $\mathrm{GuHCl}$, glucose, and glycine. Phosphate-buffered saline (PBS) pH 7.4 was used as the control. For each test solute, a solution was prepared containing $280 \mathrm{mM}$ sorbitol, $140 \mathrm{mM} \mathrm{GuHCl}, 280 \mathrm{mM}$ glucose, or $280 \mathrm{mM}$ glycine with $20 \mathrm{mM}$ HEPES, $\mathrm{pH}$ 7.4. All solutions were filter sterilized with $0.2 \mu \mathrm{m}$ membrane filters. The procedure was carried out at the room temperature $\left(25-27^{\circ} \mathrm{C}\right)$. Asynchronous parasite culture was first harvested by centrifugation at $600 \times g$ for $5 \mathrm{~min}$. The packed RBCs $(0.25 \mathrm{~mL})$ was resuspended in $10 \mathrm{~mL}$ of each test solution by gently mixing with a pipette and incubated for $20 \mathrm{~min}$. Cells were then centrifuged
$(600 \times g$ for $5 \mathrm{~min})$ and the supernatant was removed. The cell pellet was washed twice with $10 \mathrm{~mL}$ of RPMI1640 incomplete medium before placing into culture. For thin blood smear preparation, the pellet was adjusted to 40-50\% haematocrit with complete medium. The blood smears were prepared, fixed with methanol, and stained with $10 \%$ Giemsa. The percent parasitaemia of each stage was determined under the microscope by counting intact infected RBCs from at least of 3000 cells three times.

\section{Final GuHCl synchronization protocol for Plasmodium knowlesi in vitro culture}

1. Harvest cells by centrifugation at $600 \times g$ for $5 \mathrm{~min}$ at room temperature.

2. Resuspend cell pellet in at least $20 \mathrm{X}$ volume of $140 \mathrm{mM} \mathrm{GuHCl}, 20 \mathrm{mM}$ HEPES, pH 7.4 and incubate at room temperature for $20 \mathrm{~min}$.

3. Collect cells by centrifugation at $600 \times g$ for $5 \mathrm{~min}$ at room temperature and remove supernatant.

4. Wash cell pellet twice with at least 20X volume of RPMI-1640 incomplete medium.

5. Resuspend cell pellet in complete medium and return cells to culture under mixed gas.

\section{Measuring parasite multiplication after $\mathrm{GuHCl}$ treatment}

To compare the multiplication rates of ring-stage parasites after $\mathrm{GuHCl}$ treatment to those obtained by density gradient centrifugation, schizonts were first purified by centrifugation of parasite culture through 55\% Nycodenz [6]. After two washes with RPMI-1640, the parasites were placed back in culture with uninfected RBCs at $2 \%$ haematocrit for $6 \mathrm{~h}$ to allow maturation and invasion. The parasites (0-6 h old rings + remaining schizonts) were then subjected to a) $\mathrm{GuHCl}$ treatment or b) a second round of centrifugation through 55\% Nycodenz (the bottom fraction under the Nycodenz cushion now contained only rings). Rings were then harvested, washed twice with RPMI-1640, and placed back in culture. Giemsa-stained thin smears were prepared for parasitaemia determination immediately after ring selection $(0 \mathrm{~h})$ and during the next cycle $(36 \mathrm{~h})$. Percent parasitaemia was determined by counting at least $2500 \mathrm{RBCs}$, the $95 \%$ confidence interval of which calculated according to the binomial distribution.

\section{Results}

To identify small molecules that could be used to synchronize P. knowlesi culture, a few common solutes were tested for their ability to selectively induce osmotic lysis of mature parasite-infected RBCs (Fig. 1). Asynchronous $P$. knowlesi in vitro culture was incubated in 
a

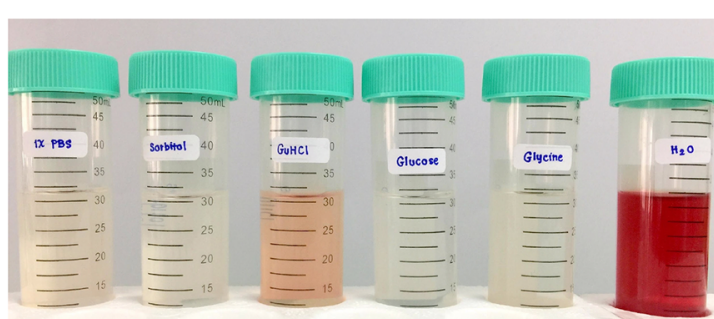

b

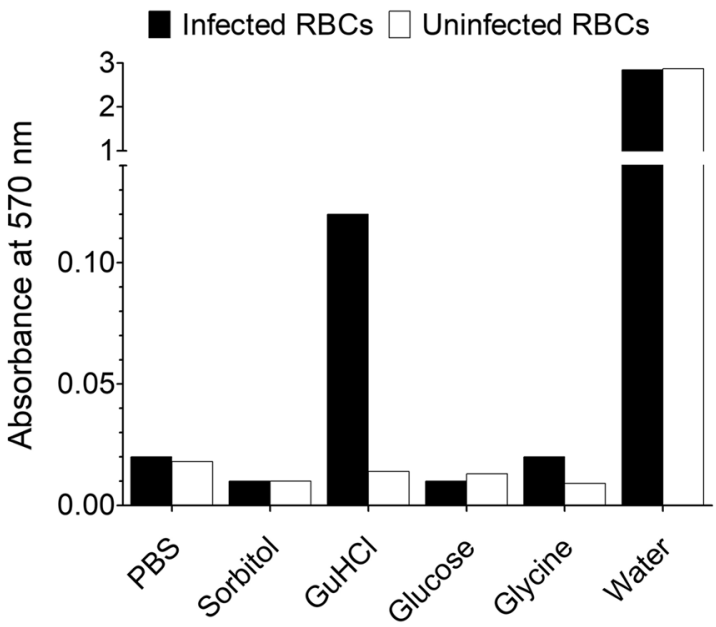

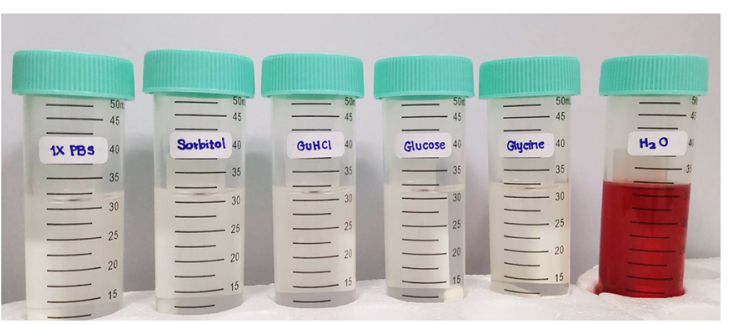

c $\square$ Ring $\square$ Trophozoite $\square$ Schizont
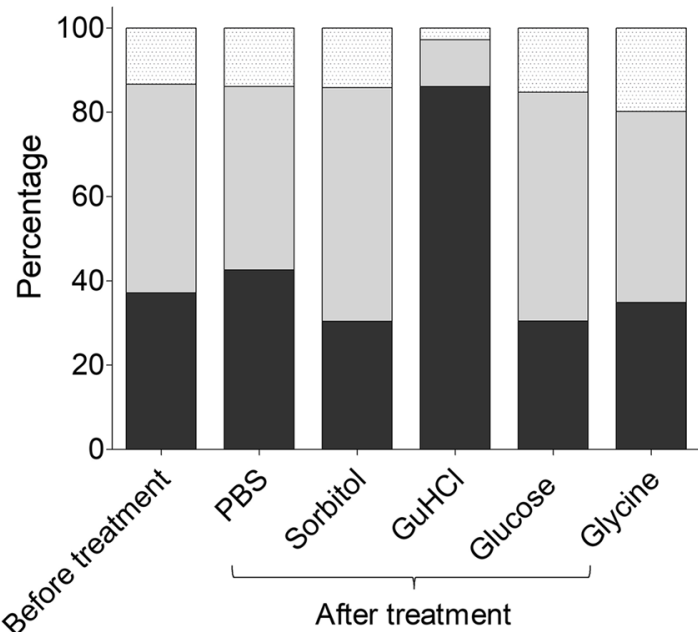

Fig. $1 \mathrm{GuHCl}$ induces lysis of trophozoite- and schizont-infected erythrocyte. a Left, supernatants of P. knowlesi-infected (1.1\% ring, 1.5\% trophozoite, $0.4 \%$ schizont) human RBC culture after treatment with PBS, sorbitol, GuHCl, glucose, glycine, or distilled water. Right, supernatant of uninfected RBCs after the same treatments. $\mathbf{b}$ Haemoglobin released, quantified by absorbance at $570 \mathrm{~nm}$. c Stage distribution of the parasite before and after each treatment. Data from a single experiment in a

HEPES-buffered solutions of sorbitol $(280 \mathrm{mM}), \mathrm{GuHCl}$ $(140 \mathrm{mM})$, glucose $(280 \mathrm{mM})$, or glycine $(280 \mathrm{mM})$ for $20 \mathrm{~min}$ at room temperature. These concentrations were chosen to keep the buffers approximately iso-osmotic. PBS and distilled water were included as negative and positive controls, respectively. Treatment with sorbitol, glucose, or glycine did not cause lysis of RBCs infected with P. knowlesi A1-H.1, whereas $\mathrm{GuHCl}$ treatment resulted in lysis of infected RBCs (Fig. 1a, b). Giemsastained blood smears clearly showed presence of all parasite stages of the asynchronous culture after sorbitol treatment, whereas in $\mathrm{GuHCl}$-treated culture the majority of remaining parasites were at the ring stage (Fig. 2). Quantification of parasite developmental stages demonstrated that $\mathrm{GuHCl}$ treatment disproportionally reduced the numbers trophozoite and schizonts (Figs. 1c, 2). Thus far, we could consistently obtain over $85 \%$ ring stage from a mixed culture.

To test whether $\mathrm{GuHCl}$ treatment is toxic to the parasites, we followed parasite development after treatment. Microscopic examination of the $\mathrm{GuHCl}$-synchronized ring culture every $2 \mathrm{~h}$ over $28 \mathrm{~h}$ revealed that the parasites developed normally and completed the intraerythrocytic cycle within $28 \mathrm{~h} \mathrm{(Fig.} \mathrm{3)} \mathrm{as} \mathrm{previously} \mathrm{reported} \mathrm{[6].} \mathrm{The}$ multiplication rate of $\mathrm{GuHCl}$ synchronized parasites was also compared to that of rings purified by density gradient centrifugation (Fig. 4). In two biological replicates out of three, the fold increases in parasitaemia of these two cell preparations were similar (Fig. 4a, b), confirming normal parasite development after $\mathrm{GuHCl}$ synchronization. However, in one replicate (Fig. 4c), parasitaemia did not increase after $\mathrm{GuHCl}$ treatment. The reason for this is still unclear, but this finding suggests that $\mathrm{GuHCl}$ may be toxic to the parasite under some circumstance.

\section{Discussion}

In this study, a method for obtaining a highly synchronous ring-stage culture of $P$. knowlesi is reported. The method is a modification of the standard sorbitol synchronization protocol of $P$. falciparum which does not work with the human adapted A1-H.1 line of P. knowlesi [15]. It was found that replacing sorbitol with $\mathrm{GuHCl}$ can achieve synchronization of this parasite. 


\section{a GuHCl treatment}
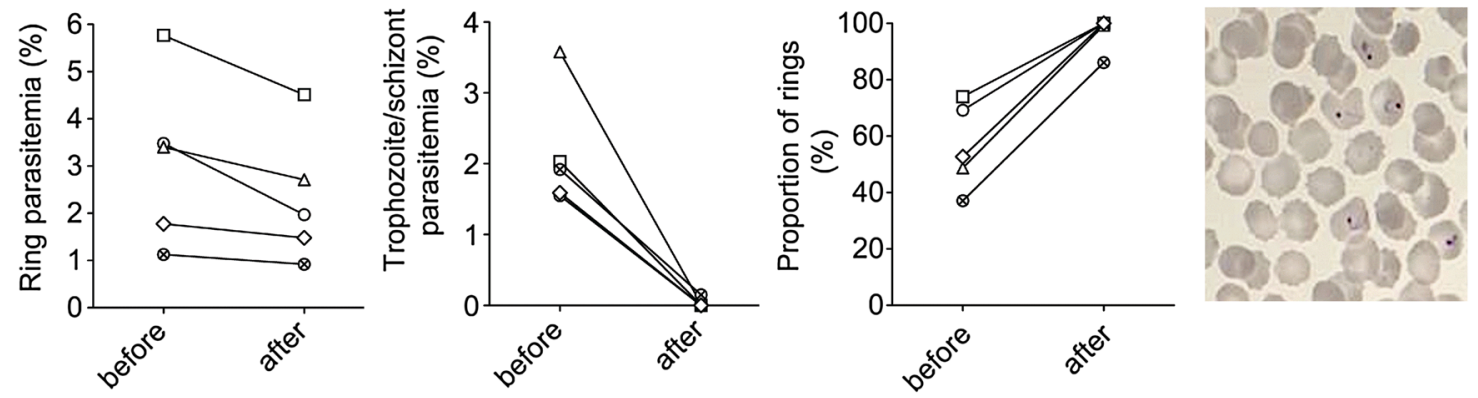

\section{b Sorbitol treatment}
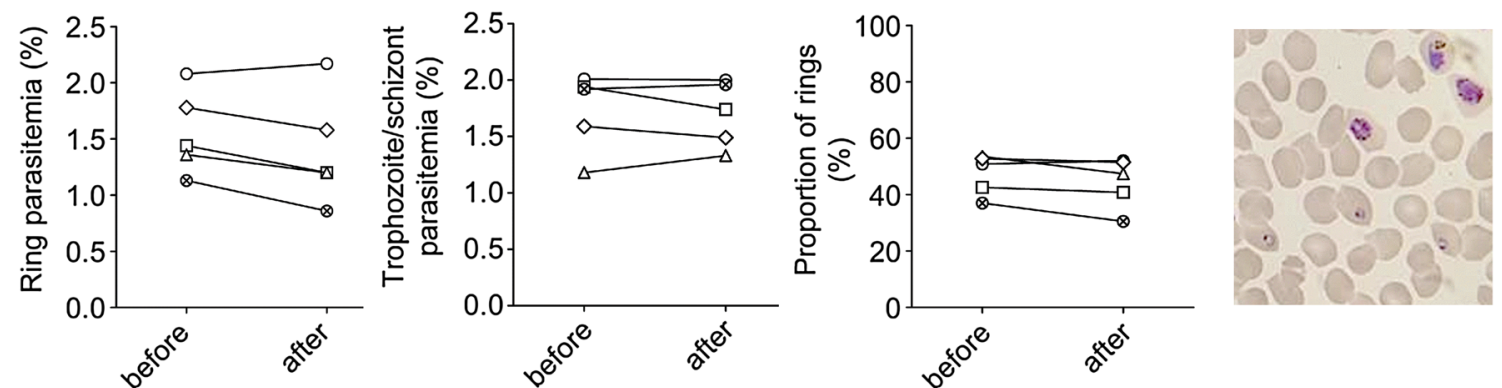

Fig. 2 Synchronization of P. knowlesi A1-H.1 parasite by GuHCl. a GuHCl treatment. b Sorbitol treatment. Left to right: ring parasitaemia before and after treatment, trophozoite and schizont parasitaemia before and after treatment, proportion of rings before and after treatment, representative Giemsa-stained thin smear of infected RBC culture after treatment. Different symbols represent different biological replicates $(N=5)$
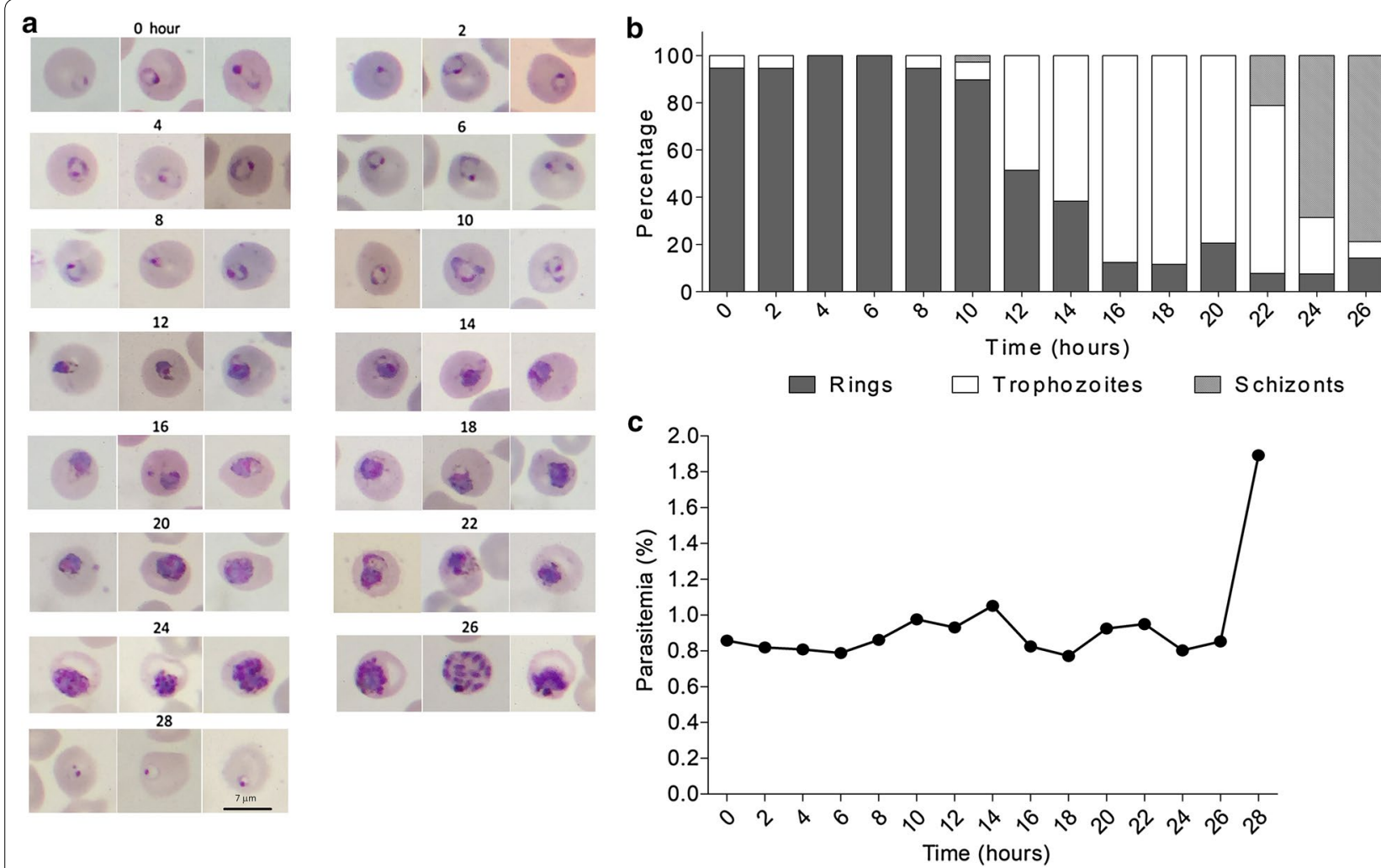

Fig. 3 Development of GuHCl synchronized P. knowlesi A1-H.1. a Giemsa-stained thin blood smears showing the morphology of the parasites during asexual growth after $\mathrm{GuHCl}$ treatment. $\mathbf{b}$ Parasite stage distribution from the same experiment. $\mathbf{c}$ Total parasitaemia every $2 \mathrm{~h}$ after synchronization from the same experiment 
The resistance of A1-H.1 to sorbitol is surprising for two reasons. Firstly, aside from a few purposefully selected P. falciparum laboratory lines [16-18], NPPs are considered conserved across Plasmodium species [14, 19-21]. Secondly, rhesus RBCs infected with $P$. knowlesi from in vitro culture are sensitive to sorbitol treatment [22]. The low sorbitol permeability of the A1-H.1 strain in human RBCs observed here may be due to mutations or epigenetic changes that occurred during the parasite's adaptation to in vitro culture in human RBCs. The prime candidates for this permeability change are the RhopH complex components whose role was previously implicated in the NPPs activity [23-25]. Alternatively, a specific human RBC protein(s) may influence NPPs function. A comparison of the NPPs properties of A1-H.1 infected rhesus and human RBCs should be able to shed light on this divergence.

Several techniques are available to enrich the Plasmodium parasites at a specific stage. Most of them were developed for P. falciparum, including physical separation based on differential density [10], temperature cycling [26], cell cycle inhibitors [27], magnetic column purification $[7,8,28]$, and Plasmion $[11,29]$. For $P$. knowlesi, density centrifugation and magnetic purification have been used to isolate mature stages $[6,30,31]$. Besides these methods, the recent report of merozoite invasion inhibition by heparin and sulfated polymers suggests that these inhibitors may also be a viable alternative for parasite synchronization [32]. The method of $\mathrm{GuHCl}$ synchronization here offers another means to obtain the ring stage. The advantage of this method is that it is simple, fast, and scalable.

Besides P. knowlesi A1-H.1, there are other lines of $P$. knowlesi that have been adapted for long-term in vitro culture. These include the original $\mathrm{H}$ strain adapted to grow in rhesus RBCs [33] and the $\mathrm{H}_{\text {hu }}$ line adapted to grow in human RBCs [5]. In this study, the $\mathrm{GuHCl}$ synchronization method was tested only with A1-H.1 grown in human RBCs. How well the method performs with the $\mathrm{H}$ and $\mathrm{H}_{\text {hu }}$ lines is currently not known, nor is the effectiveness of the method for the A1-H.1 line grown in simian RBCs. However, because high guanidinium permeability of infected RBCs has been observed even with distantly related P. falciparum [34], it is possible that the $\mathrm{GuHCl}$ synchronization method will work universally well with other $P$. knowlesi lines as well as with $P$. falciparum.

\section{Limitations}

$\mathrm{GuHCl}$ synchronization is routinely used in the authors' laboratory. While this method works well and yields healthy ring-stage parasites most of the time, it can sometimes result in parasites with a slow multiplication
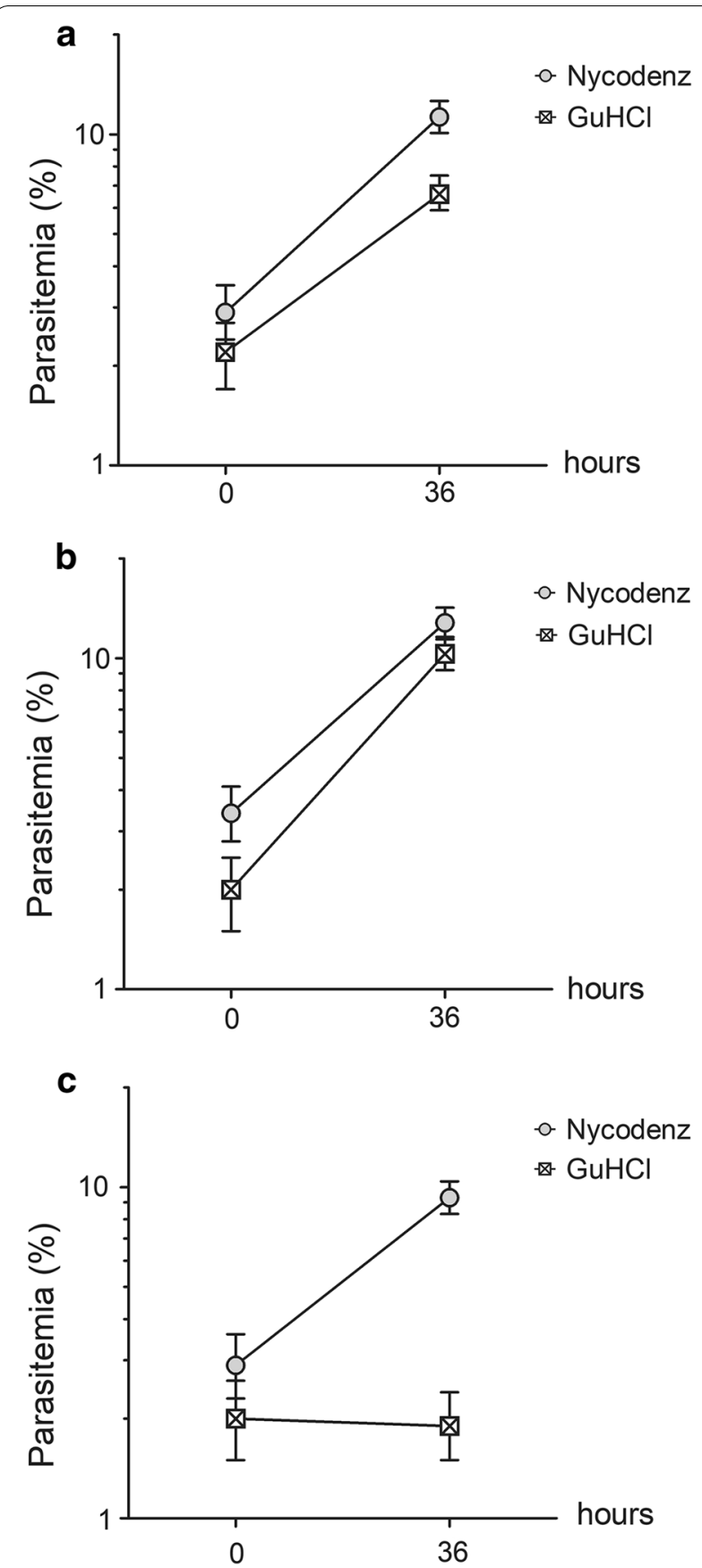

Fig. 4 Parasite multiplication following ring synchronization by GuHCl or density gradient (Nycodenz) centrifugation. Parasitaemia was determined immediately after synchronization and at $36 \mathrm{~h}$ later. a-c Represent different biological replicates (A1-H.1 cultures in different human RBCs). Error bars indicate $95 \%$ confidence intervals

rate, a risk revealed in Fig. 4c. The reason for this phenomenon is still unclear, but it may limit the utility of this method when delayed parasite growth needs to be avoided. Normal multiplication rates usually resume within one or two cycles. 


\section{Conclusions}

This study demonstrates the utility of $\mathrm{GuHCl}$ as an effective means for synchronization of the ring-stage $P$. knowlesi A1-H.1 in vitro culture in human RBCs. The failure of sorbitol to synchronize this line of parasite suggests that NPPs of this parasite line has a distinct solute permeability profile when compared to that of $P$. falciparum. The methodology described could be applied to identify a solute appropriate for osmotic synchronization of other parasite or parasite lines that may be resistant to sorbitol or guanidine treatment.

\section{Abbreviations \\ GuHCl: guanidine hydrochloride; h: hour; HEPES: (4-(2-hydroxyethyl)-1-piper- azineethanesulfonic acid; min: minute; NPPS: new permeation pathways; PBS:} phosphate-buffered saline; RBCs: red blood cells.

\section{Authors' contributions}

SN planned and conducted all experiments, analyzed data, and was a major contributor in drafting the manuscript. AC assisted in collecting the time course data after synchronization. WR guided the experiments and revised the manuscript. JS and LC guided the experiment and revised the manuscript. WN conceived of research, designed experiments, and was a major contributor in preparing the manuscript. All authors read and approved the final manuscript.

\section{Author details \\ ${ }^{1}$ Department of Molecular Tropical Medicine, Faculty of Tropical Medicine, Mahidol University, 420/6 Ratchawithi Road, Ratchathewi, Bangkok 10400, Thailand. ${ }^{2}$ Mahidol Vivax Research Unit, Faculty of Tropical Medicine, Mahidol University, 420/6 Ratchawithi Road, Ratchathewi, Bangkok 10400, Thailand. ${ }^{3}$ Department of Internal Medicine, Morsani College of Medicine, University of South Florida, Tampa, FL, USA.}

\section{Acknowledgements}

Not applicable.

\section{Competing interests}

The authors declare that they have no competing interests.

\section{Availability of data and materials}

The datasets used and/or analyzed during the current study are available from the corresponding author on reasonable request.

\section{Consent for publication}

Not applicable.

\section{Ethics approval and consent to participate}

The need for ethics approval for this study was waived by The Ethics Committee at the Faculty of Tropical Medicine, Mahidol University.

\section{Funding}

This study was supported by an Intermediate Fellowship to Public Health and Tropical Medicine from Wellcome Trust to WN (101073/Z/13Z), a student fellowship to SN (SCA-CO-2558-725-TH) from National Science and Technology Development Agency (NSTDA). AC and SN were supported by a training grant (D43TW006571) from the National Institutes of Health, USA.

\section{Publisher's Note}

Springer Nature remains neutral with regard to jurisdictional claims in published maps and institutional affiliations.

Received: 21 January 2019 Accepted: 16 April 2019

Published online: 25 April 2019

\section{References}

1. Cox-Singh J, Singh B. Knowlesi malaria: newly emergent and of public health importance? Trends Parasitol. 2008;24:406-10.

2. White NJ. Plasmodium knowlesi: the fifth human malaria parasite. Clin Infect Dis. 2008;46:172-3.

3. Millar SB, Cox-Singh J. Human infections with Plasmodium knowlesizoonotic malaria. Clin Microbiol Infect. 2015;21:640-8.

4. Singh B, Daneshvar C. Human infections and detection of Plasmodium knowlesi. Clin Microbiol Rev. 2013;26:165-84.

5. Lim C, Hansen E, DeSimone TM, Moreno Y, Junker K, Bei A, et al. Expansion of host cellular niche can drive adaptation of a zoonotic malaria parasite to humans. Nat Commun. 2013;4:1638.

6. Moon RW, Hall J, Rangkuti F, Ho YS, Almond N, Mitchell GH, et al. Adaptation of the genetically tractable malaria pathogen Plasmodium knowlesi to continuous culture in human erythrocytes. Proc Natl Acad Sci USA. 2013;110:531-6.

7. Mata-Cantero L, Lafuente MJ, Sanz L, Rodriguez MS. Magnetic isolation of Plasmodium falciparum schizonts iRBCs to generate a high parasitaemia and synchronized in vitro culture. Malar J. 2014;13:112.

8. Nam J, Huang H, Lim H, Lim C, Shin S. Magnetic separation of malariainfected red blood cells in various developmental stages. Anal Chem. 2013:85:7316-23.

9. Lambros C, Vanderberg JP. Synchronization of Plasmodium falciparum erythrocytic stages in culture. J Parasitol. 1979;65:418-20.

10. Rivadeneira EM, Wasserman M, Espinal CT. Separation and concentration of schizonts of Plasmodium falciparum by Percoll gradients. J Protozool. 1983;30:367-70.

11. Ranford-Cartwright LC, Sinha A, Humphreys GS, Mwangi JM. New synchronization method for Plasmodium falciparum. Malar J. 2010;9:170.

12. Rojas MO, Wasserman M. Effect of low temperature on the in vitro growth of Plasmodium falciparum. J Eukaryot Microbiol. 1993:40:149-52.

13. Yuan L, Hao M, Wu L, Zhao Z, Rosenthal BM, Li X, et al. Refrigeration provides a simple means to synchronize in vitro cultures of Plasmodium falciparum. Exp Parasitol. 2014;140:18-23.

14. Kutner S, Breuer WV, Ginsburg H, Aley SB, Cabantchik Zl. Characterization of permeation pathways in the plasma membrane of human erythrocytes infected with early stages of Plasmodium falciparum: association with parasite development. J Cell Physiol. 1985;125:521-7.

15. van Schalkwyk DA, Moon RW, Blasco B, Sutherland CJ. Comparison of the susceptibility of Plasmodium knowlesi and Plasmodium falciparum to antimalarial agents. J Antimicrob Chemother. 2017;72:3051-8.

16. Hill DA, Pillai AD, Nawaz F, Hayton K, Doan L, Lisk G, Desai SA. A blasticidin S-resistant Plasmodium falciparum mutant with a defective plasmodial surface anion channel. Proc Natl Acad Sci USA. 2007;104:1063-8.

17. Mira-Martinez S, Rovira-Graells N, Crowley VM, Altenhofen LM, Llinas M, Cortes A. Epigenetic switches in clag3 genes mediate blasticidin $\mathrm{S}$ resistance in malaria parasites. Cell Microbiol. 2013;15:1913-23.

18. Sharma P, Wollenberg K, Sellers M, Zainabadi K, Galinsky K, Moss E, et al. An epigenetic antimalarial resistance mechanism involving parasite genes linked to nutrient uptake. J Biol Chem. 2013;288:19429-40.

19. Ancelin ML, Parant M, Thuet MJ, Philippot JR, Vial HJ. Increased permeability to choline in simian erythrocytes after Plasmodium knowlesi infection. Biochem J. 1991;273(Pt 3):701-9.

20. Gati WP, Lin AN, Wang TI, Young JD, Paterson AR. Parasite-induced processes for adenosine permeation in mouse erythrocytes infected with the malarial parasite Plasmodium yoelii. Biochem J. 1990;272:277-80.

21. Neame KD, Homewood CA. Alterations in the permeability of mouse erythrocytes infected with the malaria parasite, Plasmodium berghei. Int J Parasitol. 1975;5:537-40.

22. Lisk G, Desai SA. The plasmodial surface anion channel is functionally conserved in divergent malaria parasites. Eukaryot Cell. 2005;4:2153-9.

23. Counihan NA, Chisholm SA, Bullen HE, Srivastava A, Sanders PR, Jonsdottir TK, et al. Plasmodium falciparum parasites deploy RhopH2 into the host erythrocyte to obtain nutrients, grow and replicate. Elife. 2017;6:e23217.

24. Ito D, Schureck MA, Desai SA. An essential dual-function complex mediates erythrocyte invasion and channel-mediated nutrient uptake in malaria parasites. Elife. 2017;6:e23485.

25. Nguitragool W, Bokhari AA, Pillai AD, Rayavara K, Sharma P, Turpin B, et al. Malaria parasite clag3 genes determine channel-mediated nutrient uptake by infected red blood cells. Cell. 2011;145:665-77. 
26. Haynes JD, Moch JK. Automated synchronization of Plasmodium falciparum parasites by culture in a temperature-cycling incubator. Methods Mol Med. 2002;72:489-97.

27. Naughton JA, Bell A. Studies on cell-cycle synchronization in the asexual erythrocytic stages of Plasmodium falciparum. Parasitology. 2007;134:331-7

28. Ahn SY, Shin MY, Kim YA, Yoo JA, Kwak DH, Jung YJ, et al. Magnetic separation: a highly effective method for synchronization of cultured erythrocytic Plasmodium falciparum. Parasitol Res. 2008:102:1195-200.

29. Lelievre J, Berry A, Benoit-Vical F. An alternative method for Plasmodium culture synchronization. Exp Parasitol. 2005;109:195-7.

30. Amir A, Russell B, Liew JW, Moon RW, Fong MY, Vythilingam I, et al. Invasion characteristics of a Plasmodium knowlesi line newly isolated from a human. Sci Rep. 2016;6:24623.
31. Moraes Barros RR, Gibson TJ, Kite WA, Sa JM, Wellems TE, Comparison of two methods for transformation of Plasmodium knowlesi: direct schizont electroporation and spontaneous plasmid uptake from plasmid-loaded red blood cells. Mol Biochem Parasitol. 2017;218:16-22.

32. Lyth O, Vizcay-Barrena G, Wright KE, Haase S, Mohring F, Najer A, et al. Cellular dissection of malaria parasite invasion of human erythrocytes using viable Plasmodium knowlesi merozoites. Sci Rep. 2018;8:10165.

33. Kocken $\mathrm{CH}, \mathrm{Ozw}$ ara $\mathrm{H}$, van der Wel A, Beetsma AL, Mwenda JM, Thomas AW. Plasmodium knowlesi provides a rapid in vitro and in vivo transfection system that enables double-crossover gene knockout studies. Infect Immun. 2002;70:655-60.

34. Bokhari AA, Mita-Mendoza NK, Fuller A, Pillai AD, Desai SA. High guanidinium permeability reveals dehydration-dependent ion selectivity in the plasmodial surface anion channel. Biomed Res Int. 2014;2014:741024.
Ready to submit your research? Choose BMC and benefit from:

- fast, convenient online submission

- thorough peer review by experienced researchers in your field

- rapid publication on acceptance

- support for research data, including large and complex data types

- gold Open Access which fosters wider collaboration and increased citations

- maximum visibility for your research: over $100 \mathrm{M}$ website views per year

At BMC, research is always in progress.

Learn more biomedcentral.com/submissions 